\title{
Maternal obesity and risk of Down syndrome in the offspring
}

Eric Hildebrand, Bengt Källén, Ann Josefsson, Tomas Gottvall and Marie Blomberg

\section{Linköping University Post Print}

\section{Tweet}

N.B.: When citing this work, cite the original article.

Original Publication:

Eric Hildebrand, Bengt Källén, Ann Josefsson, Tomas Gottvall and Marie Blomberg, Maternal obesity and risk of Down syndrome in the offspring, 2014, Prenatal Diagnosis, (34), 4, 310-315.

http://dx.doi.org/10.1002/pd.4294

Copyright: Wiley http://eu.wiley.com/WileyCDA/

Postprint available at: Linköping University Electronic Press

http://urn.kb.se/resolve?urn=urn:nbn:se:liu:diva-103996 


\section{Maternal obesity and risk of Down syndrome in the offspring}

Obesity and Down syndrome

2,818 words

1 table

5 figures

Eric Hildebrand $\mathrm{MD}^{1}$, Bengt Källén Prof ${ }^{2}$ Ann Josefsson $\mathrm{MD}, \mathrm{PhD}^{1}$, Tomas Gottvall $\mathrm{MD}, \mathrm{PhD}^{1}$

Marie Blomberg MD, $\mathrm{PhD}^{1}$

${ }^{1}$ Department of Clinical and Experimental Medicine, Linköping University, Department of Obstetrics and Gynecology, County Council of Östergötland, Linköping, Sweden.

${ }^{2}$ Tornblad Institute, University of Lund, Lund, Sweden.

\section{Corresponding author:}

Dr. Eric Hildebrand

Department of Obstetrics and

Gynecology

SE-581 85 Linköping

Sweden eric.hildebrand@lio.se

Phone: +46101030000 
FORSS, the Southeast Region medical research council, financially supported the study. Involvement in study design, data collection, data analysis, manuscript preparation and/or publication decisions: None.

This submission contains original unpublished work and is not being submitted for publication elsewhere at the same time.

Potential conflicts of interest: None declared.

Bulleted statements:

What's already known about this topic?

- A combination of Ultrasound and Biochemistry is a well-studied method for risk-assessment of fetal aneuploidy

What does this study add?

- ; Maternal obesity in very early pregnancy is associated with increased risk for Down syndrome in the newborn.

- Combined first trimester screening for aneuploidies is equally effective for all BMIcategories. 


\begin{abstract}
Objective: To determine if maternal obesity is associated with an increased risk of Down syndrome in the offspring and whether the risk estimates for trisomy 21 based on combined screening is affected by maternal Body Mass Index (BMI).
\end{abstract}

Method: Study group I consisted of a Nation-wide cohort of 1,568,604 women giving birth; outcome was infants born with Down syndrome. Adjustment was made for maternal age. Study group II consisted of 10,224 women undergoing $1^{\text {st }}$ trimester combined screening. Outcome was risk assessment for Down syndrome. All women were divided into six BMI groups and outcomes were evaluated over the BMI strata with BMI 18.5-24.9 as reference and correcting for maternal age.

Results: Obese women had an increased risk for giving birth to an infant with Down syndrome compared to normal weight women, BMI 30-34.9 OR 1.31 (95\% CI 1.10-1.55), BMI 35-39.9 OR 1.12 (95\%CI 0.82-1.53), BMI $\geq 40$ OR 1.56 (95\%CI 1.00-2.43). The observed and the expected numbers of women with a risk of Down syndrome $>1 / 300$ based on $1^{\text {st }}$ trimester combined screen and maternal age were similar in each BMI group.

Conclusion: Maternal obesity seems to increase the risk for Down syndrome births. The risk estimate for Down syndrome with $1^{\text {st }}$ trimester combined screening is unaffected by BMI. 


\section{Introduction}

Maternal obesity is associated with congenital birth defects, including an increased risk for neural tube defects, congenital heart defects and orofacial clefts in the offspring ${ }^{1-3}$. No association has been described, however, between maternal pre-pregnancy obesity and the prevalence of Down syndrome in offspring. Miscarriage may occur more often among obese women ${ }^{4}$. Analysis of karyotype in first trimester miscarriages showed that obese women were less likely to have aneuploidy miscarriages than normal weight women ${ }^{5,6}$.

The screening procedures for detecting Down syndrome offered to pregnant women have changed over the last decades. In the past, and probably still in many countries, amniocentesis has been the sole method used, mainly offered to women above 35 years of age. At present in many countries women in early pregnancy are instead offered combined screening with ultrasound and biochemistry, with measurement of fetal nuchal translucency (NT), maternal serum free $\beta$-human chorionic gonadotropin $(\beta-\mathrm{hCG})$ and pregnancy-associated plasma protein-A (PAPP-A), to receive their individual risk estimate for trisomy $21^{7}$. In the algorithm described by the Fetal Medicine Foundation (FMF) in London adjustments in the measured maternal $\beta$-hCG and PAPP-A are made for maternal weight ${ }^{8,9}$.

High maternal body mass index (BMI) adversely affects the ability to visualize fetal structures by ultrasound in the second trimester ${ }^{10,11}$ and it appears that maternal obesity also has implications in early pregnancy for the ultrasound measurements ${ }^{12}$. NT was found to be thicker in the fetuses of obese women $(\mathrm{BMI} \geq 30)$ than in the fetuses carried by normal weight women $^{13}$; However this was seen as having little or no clinical relevance ${ }^{14-16}$ In addition it has been found that as maternal BMI increases, the time required to obtain NT measurements and the failure rate increase ${ }^{17}$.

The objective of this study was to determine if maternal obesity is associated with an increased risk of Down syndrome in the offspring and whether the risk estimates for trisomy 
21 from the screening procedure offered in early pregnancy varies over the BMI strata. 


\section{Methods}

For the purpose of this study two study populations were used, referred to as study group I and II.

Study group I - population-based study of risk of Down syndrome births

This study population consisted of 1,568,604 women who had given birth in Sweden from January 1, 1995 through December 31, 2010. They were identified using the Swedish Medical Birth Register ${ }^{18}$. Medical and other data on almost all (98-99\%) deliveries in Sweden are listed in the register. From 1995 until June 2008 the register included stillbirths after 28 weeks gestation and from July 2008 until 2010 all stillbirths after 22 weeks gestation were included. The register is based on copies of the standardized medical record forms completed at the antenatal health care centers at the start of prenatal care, usually in gestational week 10-12, records from the delivery units, and records from the pediatric examination of the newborn. Ninety per cent of women present themselves to the antenatal health care center during the first trimester of their pregnancy. The system is identical throughout Sweden. A detailed description and validation of the register content is available ${ }^{18}$. There is no nation-wide register on terminated pregnancies in Sweden with data on Social Security Number available, which make individual identification impossible.

The study period extended over 16 years and prenatal screening procedures available for pregnant women in Sweden have changed during these 16 years. The screening procedure for Down syndrome in Sweden that was offered between 1995 and 2004 was amniocentesis and this procedure was only offered, with few exclusions, to women aged 35 or older. From 2005 to 2010 the prenatal screening procedures for Down syndrome were a mixture of amniocentesis based on maternal age, risk assessment based on NT measurements and combined ultrasound and biochemistry. 
Midwives measured maternal weight and height and recorded values in a standardized form at the woman's first visit to the antenatal health care center. Body mass index (BMI, $\mathrm{kg} / \mathrm{m}^{2}$ ) was then calculated. Women were grouped into six categories of BMI: underweight $(<18.5)$, normal weight (18.5-24.9), overweight (25-29.9), obese class I (30-34.9), obese class II (3539.9) and obese class III ( $\geq 40)$ according to World Health Organization (WHO) classification ${ }^{19}$. Obesity class III is equivalent to morbidly obese in this study. The primary outcome in this first study population was infants born with Down syndrome. The identification of infants with Down syndrome was based on information from three sources: the Medical Birth Register, the Birth Defect Register (previously called the Register of Congenital Malformations) which includes reports from cytogenetic laboratories, and the Hospital Discharge Register (part of the Patient Register) ${ }^{18,20,21}$. Together they make it possible to identify nearly all cases.

Maternal age (1-year classes) is an important confounding factor and was included in the adjusted analyses. Adjusted odds ratios (OR) were determined using the Mantel-Haenszel technique $^{22}$. Estimates of $95 \%$ confidence intervals (95\%CI) were made with a test-based method, based on the Mantel-Haenszel chi-square ${ }^{22}$

Study group II - effect of BMI on risk estimates during pregnancy. This population consisted of 10,224 women who had undergone prenatal screening for trisomy 21 between April 12009 and December 31 2011, using an algorithm employing a combination of maternal age, NT, $\beta$-hCG and PAPP-A based on the formulas derived by the Fetal Medicine Foundation in London ${ }^{7}$. In the algorithm the estimates of PAPP-A and $\beta$-hCG were adjusted for maternal weight (but not BMI).

Data were retrieved from a local clinical database (Astraia, Germany) at Linkoping University hospital, a tertiary referral center for fetal medicine. The following variables were exported from the Astraia database: maternal weight, calculated risk for trisomy 21, 
gestational day when the ultrasound examination was performed, NT thickness, maternal age at the ultrasound examination, PAPP-A multiples of the median (MoM), and free $\beta$-hCG MoM. During the study period women with a risk for trisomy 21 greater than $1 / 300$ were offered amniocentesis.

Data on maternal height were not available in the Astraia database and were therefore retrieved from the antenatal care center medical records and linked to the Astraia data set using the personal identification number held by everyone living in Sweden. The women were classified in six BMI classes as described above concerning the first study population. Outcomes estimated over the maternal BMI strata were risk assessments for Down syndrome, NT thickness, PAPP-A MoM and free $\beta$-hCG MoM. The analyses of the variables intend to study a normal population therefore cases with a risk estimate $>1 / 300$ (where most of the cases of Down syndrome could be expected) were excluded to avoid bias of a potential association between maternal BMI and Down syndrome.

Medians with 95\% CI were estimated using Analyze-it software (Analyze-it Software, Ltd, Leads, UK). In order to compare risk estimates in different BMI classes, the Wilcoxon nonparametric method was used because of the extreme skewness of data. For each maternal age class (one year) the rank sums of cases in a specific BMI class were compared with the rank sum of the reference BMI class (BMI 18.5-24.9) and the rank sum T of the former was determined with its error. The mean for these $\mathrm{T}$ values with its error was compared with zero. Zero would indicate no difference.

Ethics: The Regional Ethical Review Board in Linköping has approved the study (2013/4-31). 


\section{Results}

\section{Study group I}

The overall crude risk of having an infant with Down syndrome during the study period was 1.31 per 1000 births. The number of cases of Down syndrome, total population and prevalence of Down syndrome for each BMI category was; 20/19,241 (1.04/1,000) for underweight women, 1,041/863,493 (1.21/1,000) for normal weight women, 471/333,658 $(1.41 / 1,000)$ for overweight women, $166 / 103,402(1.61 / 1,000)$ for obesity class I, 42/30,425 $(1.38 / 1,000)$ for obesity class II and 20/10,336 $(1.93 / 1,000)$ for obesity class III $(\geq 40)$. Maternal BMI could not be calculated for $13.3 \%$ of the women due to missing data on maternal height and/or weight. The group with unknown BMI had a crude risk of having an infant with Down syndrome almost the same as for those with known BMI $(1.42 / 1,000)$. After adjustment for maternal age distribution and comparison with the normal BMI group, the OR among women with unknown BMI was 1.13, not quite statistically significant (95\% CI 0.991.29).

The prevalence of obesity $(\mathrm{BMI} \geq 30)$ was $10.6 \%$ distributed in obesity class I-III as follows: class I $7.6 \%$, class II $2.2 \%$ and class III $0.8 \%$. Obese women (BMI $\geq 30$ ) had a greater risk for giving birth to an infant with Down syndrome than did normal-weight women, OR 1.28 (95\% CI 1.12-1.48) after adjustment for maternal age (one year classes).

Figure 1 shows the risk for Down syndrome according to maternal BMI in study group I from the Swedish Medical Birth Registry. There is a clear trend of a moderately increased risk with increasing BMI .

Age-adjusted ORs over the BMI strata were also analyzed for 1995-2004 and for 2005-2010 separately (data not shown) and no major differences were found.

Study group II 
The prevalence of obesity was $12.9 \%$; the corresponding rates for obesity class I-III were; class I $9.0 \%$, class II $3.0 \%$ and class III $1.0 \%$. Study group II had a slightly higher prevalence of obesity than women in the nationwide study group I. The risk estimates for Down syndrome show an extremely skewed distribution. Figure 2 shows the median risk in the first trimester for each BMI class, expressed as the risk per 10,000 pregnancies with the median for the normal BMI class marked as a dotted horizontal line. Women with a calculated trisomy 21 risk of $>1 / 300$ were excluded. There is a clear trend of an increased risk with increasing BMI. Underweight women had the lowest risk for Down syndrome and morbidly obese women the highest risk estimate.

Mean measured NT thickness increased with increasing maternal BMI (Figure 3), without adjustment for maternal age and with exclusion of women with a calculated risk of $>1 / 300$. No significant difference was seen in gestational age at examination between the different BMI-groups.

The levels of PAPP-A MoM and free $\beta$-hCG MoM are adjusted for maternal weight in the formula for calculating the risk of Down syndrome. When comparing the levels of weightadjusted PAPP-A MoM and free $\beta$-hCG MoM between the maternal BMI strata (Figure 4) no significant effect for PAPP-A or $\beta$-hCG was observed. Women with a calculated trisomy 21 risk of $>1 / 300$ were excluded.

Since the risk for trisomy 21 markedly increases with woman's age and the BMI distribution also varies with age, adjustment for maternal age was made. Figure 5 shows the mean risk estimate for each maternal age (1-year) for the normal BMI class (18.5-24.9) and for overweight and obesity class I and II+III. A clear-cut difference is seen for obese women and at ages greater than 35 years, with higher adjusted risk for Down syndrome in older obese compared with lighter weight women. 
In order to see if these effects were statistically significant, we used the Wilcoxon nonparametric test and compared the risk for obese women and normal-BMI women with a T-test (rank order test) within each maternal age. We calculated the mean $\mathrm{T}$ value for all ages and compared with 0 which was the expected value if no difference existed. For both BMI group 30-34.9 ( mean $\mathrm{T}=0.81 \pm 0.28, \mathrm{z}=2.89, \mathrm{P}=0.006)$ and $\mathrm{BMI}$ group $\geq 35($ mean $\mathrm{T}=0.40 \pm 0.27$, $\mathrm{z}=1.50, \mathrm{p}=0.13)$ there was a higher risk for obese women than for those with normal BMI., but the difference was not statistically significant The risk limit above which amniocentesis was offered during the study period was a risk for trisomy 21 higher than 1/300. Table 1 show that the percentages of women in each BMI class with a risk estimate $>1 / 300$ differed according to BMI class. Since the age distribution differed between the BMI classes, we calculated the expected number of such cases in each BMI class, adjusting for maternal age (one year class). The expected number of cases with a $>1 / 300$ risk within each BMI class was thus estimated from the age-specific risk for Down syndrome multiplied with the number of individuals in that BMI and age class. As is seen in Table 1, the observed and expected numbers were very close, suggesting that after controlling for maternal age, the rate of Down syndrome risk $>1 / 300$ was not affected by maternal BMI. 


\section{Discussion}

This large population-based cohort study based on Swedish Medical Birth Register data showed that the risk of giving birth to an infant with Down syndrome was $28 \%$ higher among obese women compared to normal weight women after adjustment for one-year maternal age. This could be due to an increased risk of having a trisomy 21 conception related to obesity or could be because obesity makes prenatal detection of trisomy 21 more difficult. When evaluating study group II a moderate effect of maternal obesity on the NT thickness measurement was found: the NT thickness increased with increasing BMI, which increases the risk estimate for Down syndrome. Both $\beta$-hCG and PAPP-A are adjusted for maternal weight in the algorithm. However, the proportion of women who had a risk estimate $>1 / 300$ (which was the value that had to be exceeded for amniocentesis to be offered) increased with BMI but was completely explained by the distribution of maternal age within each BMI class. Thus there is no evidence that any effects of BMI on the components of first trimester combined screen affects the risk estimate past the critical level. These findings are in accordance with earlier studies ${ }^{7,13-16}$. It should be emphasized that the risk estimates from the combined screening studies (Study group II) have a minor effect on the results from Study group I as such screening was only seldom performed in the whole country during the study period. If a high BMI is associated with an inadequate prenatal identification of trisomy 21 , the explanation must be sought in other factors. One possibility is that obesity may affect prenatal detection of structural fetal defects or findings of soft markers associated with Down syndrome in the second trimester of pregnancy, leading to lower rates of amniocentesis and trisomy 21 diagnosis in the midtrimester ${ }^{11}$. It appears that it is more difficult to detect structural fetal defects in obese women at the second trimester screening than in women of normal weight ${ }^{12,23,10}$. A higher detection rate of anomalies among lean and normal-weight women could provide the reason 
for amniocentesis and abnormal results at amniocentesis could lead to higher rates of termination of pregnancy than among obese women. A systematic search of US Englishlanguage articles (1995-2011) by Natoli reporting data for pregnancies with definitive prenatal diagnosis of Down syndrome, showed that termination rates varied with maternal age, gestational age, and maternal ethnicity ${ }^{24}$. However maternal BMI in relation to termination of Down syndrome pregnancies was not evaluated in the Natoli study. Obesity is linked to low socioeconomic status and a second possibility is that obese women may attend antenatal care to a lesser extent than normal weight women ${ }^{25,26}$. The socioeconomic status of the women may also affect either the woman's acceptance of an offer of prenatal diagnosis or the woman's acceptance of amniocentesis if the risk of Down syndrome is increased. The proportion of women who were offered but declined prenatal screening with combined ultrasound and biochemistry during the study period was $18 \%$. Unfortunately the BMIs of these women was not recorded. In our cohort of screened women with a risk for Down syndrome $>1 / 300$ there were no differences in mean weight between women accepting and declining amniocentesis.

If the above factors do not explain the association between high maternal BMI and increased risk for Down syndrome infants, we should consider an effect of obesity on the risk for the non-disjunction process underlying Trisomy 21. Some studies have suggested that nutritional factors with insufficient folic acid supplementation could affect the Down risk but a recent large study found little evidence for this - only at a maternal age $\geq 35$ and a meiosis II error was an association seen, but this could be the result of multiple testing ${ }^{27}$. The advantage of population-based register studies is the large number of individuals available for evaluation, which gives high statistical power. The availability of a sufficient number of study subjects made it possible to evaluate the three subgroups of obesity suggested by WHO, obesity class I-III. The drawback could be the large number of health 
care units involved, which could result in a difference in the assessment of risk of Down syndrome infants, but it seems unlikely that such variability is related to maternal BMI. Finally the possibility exists that there is a real direct effect of obesity or conditions associated with obesity and the origin of chromosomal non-disjunction but the possible mechanisms behind such an effect are not yet known.

\section{Conclusion}

Prenatal screening for Down syndrome with combined ultrasound and biochemistry offered to women in the first trimester seems equally effective irrespective of maternal BMI.

Maternal obesity increases the risk for Down syndrome in the offspring, and the effect is most prominent at older maternal ages. 


\section{References}

1 Blomberg MI, Kallen B. Maternal obesity and morbid obesity: the risk for birth defects in the offspring. Birth Defects Res A Clin Mol Teratol 2010; 88:35-40.

2 Stothard KJ, Tennant PW, Bell R, et al. Maternal overweight and obesity and the risk of congenital anomalies: a systematic review and meta-analysis. Jama 2009; 301:63650 .

3 Watkins ML, Rasmussen SA, Honein MA, et al. Maternal obesity and risk for birth defects. Pediatrics 2003; 111:1152-8.

$4 \quad$ Metwally M, Ong KJ, Ledger WL, et al. Does high body mass index increase the risk of miscarriage after spontaneous and assisted conception? A meta-analysis of the evidence. Fertil Steril 2008; 90:714-26.

5 Kroon B, Harrison K, Martin N, et al. Miscarriage karyotype and its relationship with maternal body mass index, age, and mode of conception. Fertil Steril 2011; 95:1827-9.

6 Landres IV, Milki AA, Lathi RB. Karyotype of miscarriages in relation to maternal weight. Hum Reprod 2010; 25:1123-6.

7 Kagan KO, Etchegaray A, Zhou Y, et al. Prospective validation of first-trimester combined screening for trisomy 21 . Ultrasound in obstetrics \& gynecology : the official journal of the International Society of Ultrasound in Obstetrics and Gynecology 2009; 34:148.

8 Spencer K, Bindra R, Nicolaides KH. Maternal weight correction of maternal serum PAPP-A and free beta-hCG MoM when screening for trisomy 21 in the first trimester of pregnancy. Prenat Diagn 2003; 23:851-5.

9 Kagan KO, Wright D, Spencer K, et al. First-trimester screening for trisomy 21 by free beta-human chorionic gonadotropin and pregnancy-associated plasma protein-A: impact of maternal and pregnancy characteristics. Ultrasound in obstetrics \& gynecology : the official journal of the International Society of Ultrasound in Obstetrics and Gynecology 2008; 31:493-502.

10 Thornburg LL, Miles K, Ho M, et al. Fetal anatomic evaluation in the overweight and obese gravida. Ultrasound Obstet Gynecol 2009; 33:670-5.

11 Aagaard-Tillery KM, Flint Porter T, Malone FD, et al. Influence of maternal BMI on genetic sonography in the FaSTER trial. Prenatal Diagnosis 2010; 30:14-22.

12 Hendler I, Blackwell SC, Bujold E, et al. The impact of maternal obesity on midtrimester sonographic visualization of fetal cardiac and craniospinal structures. Int J Obes Relat Metab Disord 2004; 28:1607-11.

13 Rode L, Ekelund C, Pedersen NG, et al. Maternal smoking, obesity and male fetal sex predispose to a large nuchal translucency thickness in healthy fetuses. Fetal Diagn Ther 2011; 29:201-7.

14 Krantz DA, Hallahan TW, Macri VJ, et al. Maternal weight and ethnic adjustment within a first-trimester Down syndrome and trisomy 18 screening program. Prenatal Diagnosis 2005; 25:635-40.

15 Sahota DS, Leung TY, Fung TY, et al. Medians and correction factors for biochemical and ultrasound markers in Chinese women undergoing first-trimester screening for trisomy 21. Ultrasound in obstetrics \& gynecology : the official journal of the International Society of Ultrasound in Obstetrics and Gynecology 2009; 33:387-93.

16 Cowans NJ, Spencer K. The relationship between maternal body mass, smoking status and ethnicity and first trimester nuchal translucency thickness. Prenatal Diagnosis $2011 ; 31: 446-9$. 
17 Gandhi M, Fox NS, Russo-Stieglitz K, et al. Effect of increased body mass index on first-trimester ultrasound examination for aneuploidy risk assessment. Obstetrics and gynecology 2009; 114:856-9.

18 National Board of Health and Welfare. Centre for Epidemiology. The Swedish Medical Birth Register - a summary of content and quality. 2003.

19 Obesity: preventing and managing the global epidemic. Report of a WHO consultation. World Health Organ Tech Rep Ser 2000; 894:i-xii, 1-253.

20 National Board of Health and Welfare. Centre for Epidemiology. Registration of congenital malformations in Swedish health registers. 2004.

21 National Board of Health and Welfare. Centre for Epidemiology. In-patient diseases in Sweden 1987-2011 (Article no. 2012-10-18)

2012.

22 Mantel N HW. Statistical aspects of the analyses of data from retrospective studies of disease. J Nat Cancer Inst 1959; 1959:719-48.

23 Hildebrand E, Gottvall T, Blomberg M. Maternal obesity and detection rate of fetal structural anomalies. Fetal Diagn Ther 2013; 33:246-51.

24 Natoli JL, Ackerman DL, McDermott S, et al. Prenatal diagnosis of Down syndrome: a systematic review of termination rates (1995-2011). Prenatal Diagnosis 2012; 32:142-53.

25 Park JH, Lee BE, Park HS, et al. Association between pre-pregnancy body mass index and socioeconomic status and impact on pregnancy outcomes in Korea. J Obstet Gynaecol Res 2011; 37:138-45.

26 James WP, Nelson M, Ralph A, et al. Socioeconomic determinants of health. The contribution of nutrition to inequalities in health. Bmj 1997; 314:1545-9.

27 Oliver TR, Tinker SW, Allen EG, et al. Altered patterns of multiple recombinant events are associated with nondisjunction of chromosome 21. Hum Genet 2012; 131:1039-46. 
Table 1. Number and percentage of women in study group II with a risk of Down syndrome $>1 / 300$ for each BMI class after adjustment for maternal one year age distribution. Expected number of cases due to maternal age is shown as a comparison.

\begin{tabular}{|l|l|l|l|l|}
\hline BMI class & Number & Total & Per cent & Expected \\
& $>1 / 300$ & number & $>1 / 300$ & number \\
\hline$<18.4$ & 8 & 206 & 3.9 & 7.4 \\
\hline $18.5-24.9$ & 286 & 5899 & 4.1 & 285.0 \\
\hline $25-29.9$ & 154 & 2799 & 5.5 & 156.5 \\
\hline $30-34.9$ & 53 & 918 & 5.7 & 52.8 \\
\hline $35-39.9$ & 18 & 304 & 5.9 & 13.9 \\
\hline$\geq 40$ & 8 & 98 & 8.2 & 7.6 \\
\hline
\end{tabular}


Figure 1: The risk of Down syndrome births in study group I from the Swedish Medical Birth Registry. Odds ratio (OR) with 95\% confidence interval (95\% CI) for Down syndrome at different BMI classes, adjusted for maternal age (one-year). Horizontal line shows reference, normal BMI.

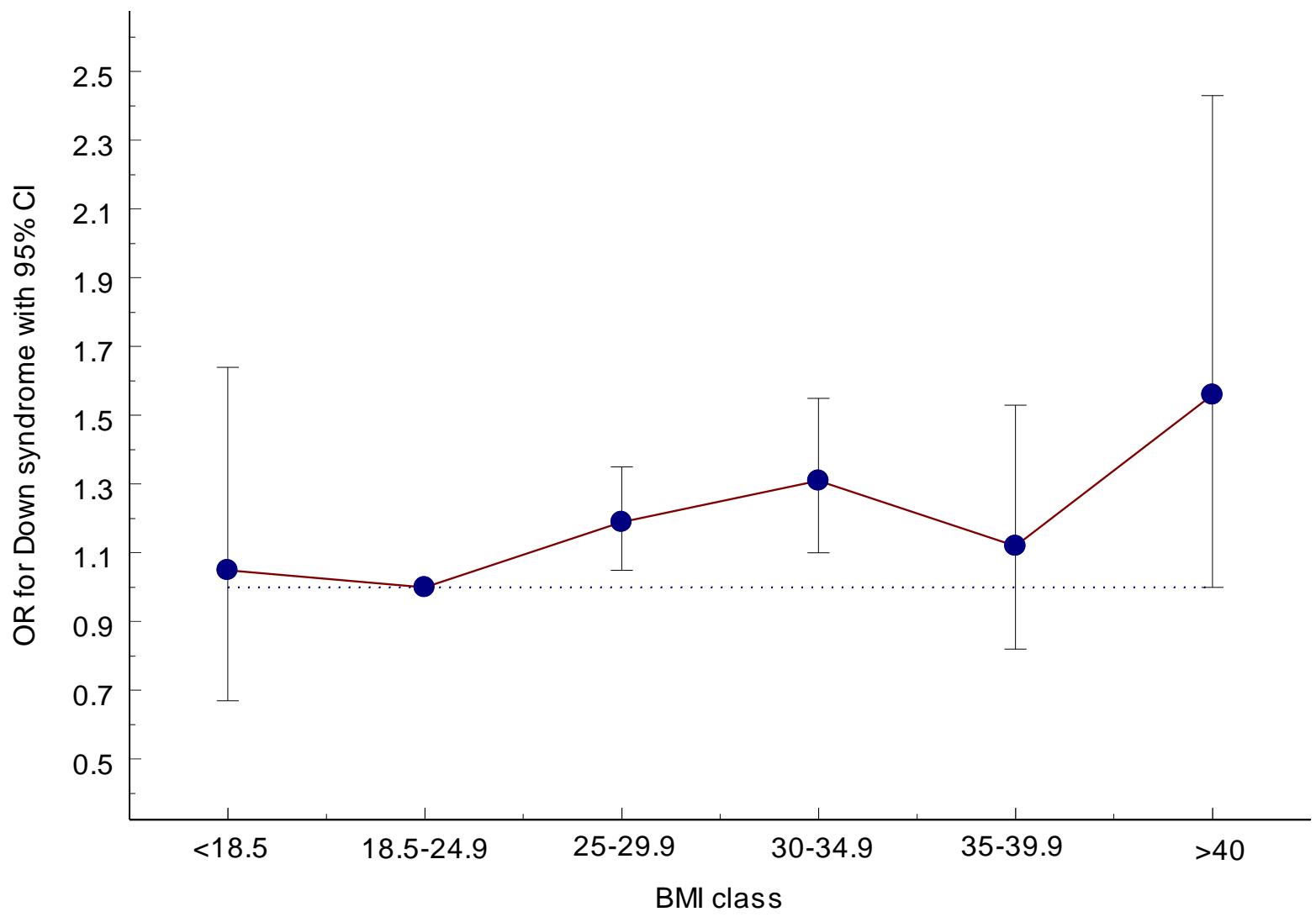


Figure 2: The risk for trisomy 21 based on study group II who had undergone prenatal screening. Median risk for trisomy 21 with 95\% confidence intervals according to woman's BMI. Women with a calculated risk $>1 / 300$ were excluded. Horizontal line shows mean for the normal BMI group (18.5-24.9).

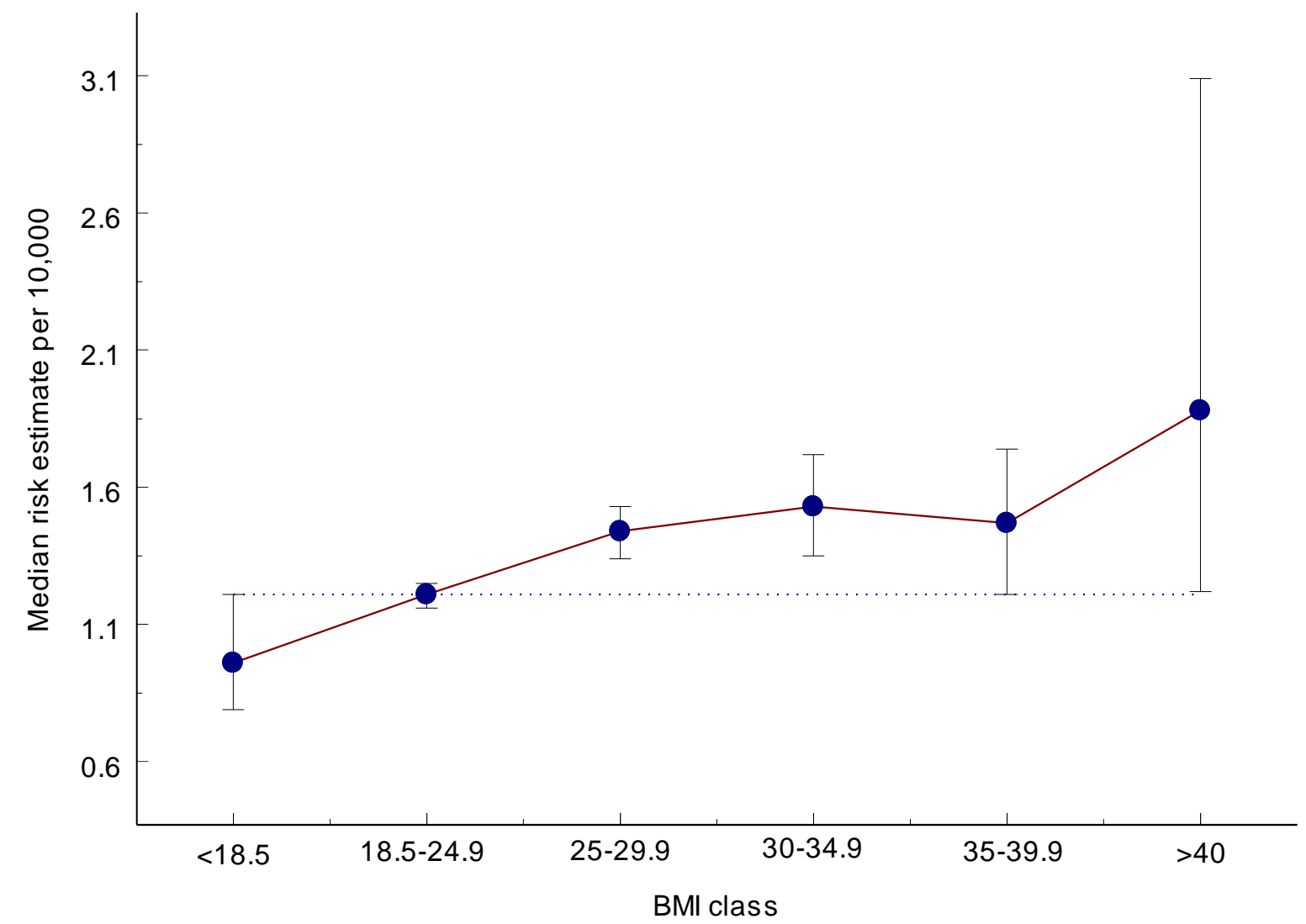


Figure 3: Means of nuchal translucency thickness (mm) according to BMI class based on study group II who had undergone prenatal screening. Cases with a trisomi 21 risk $>1 / 300$ were excluded. Horizontal line shows mean for the normal BMI group (18.5-24.9).

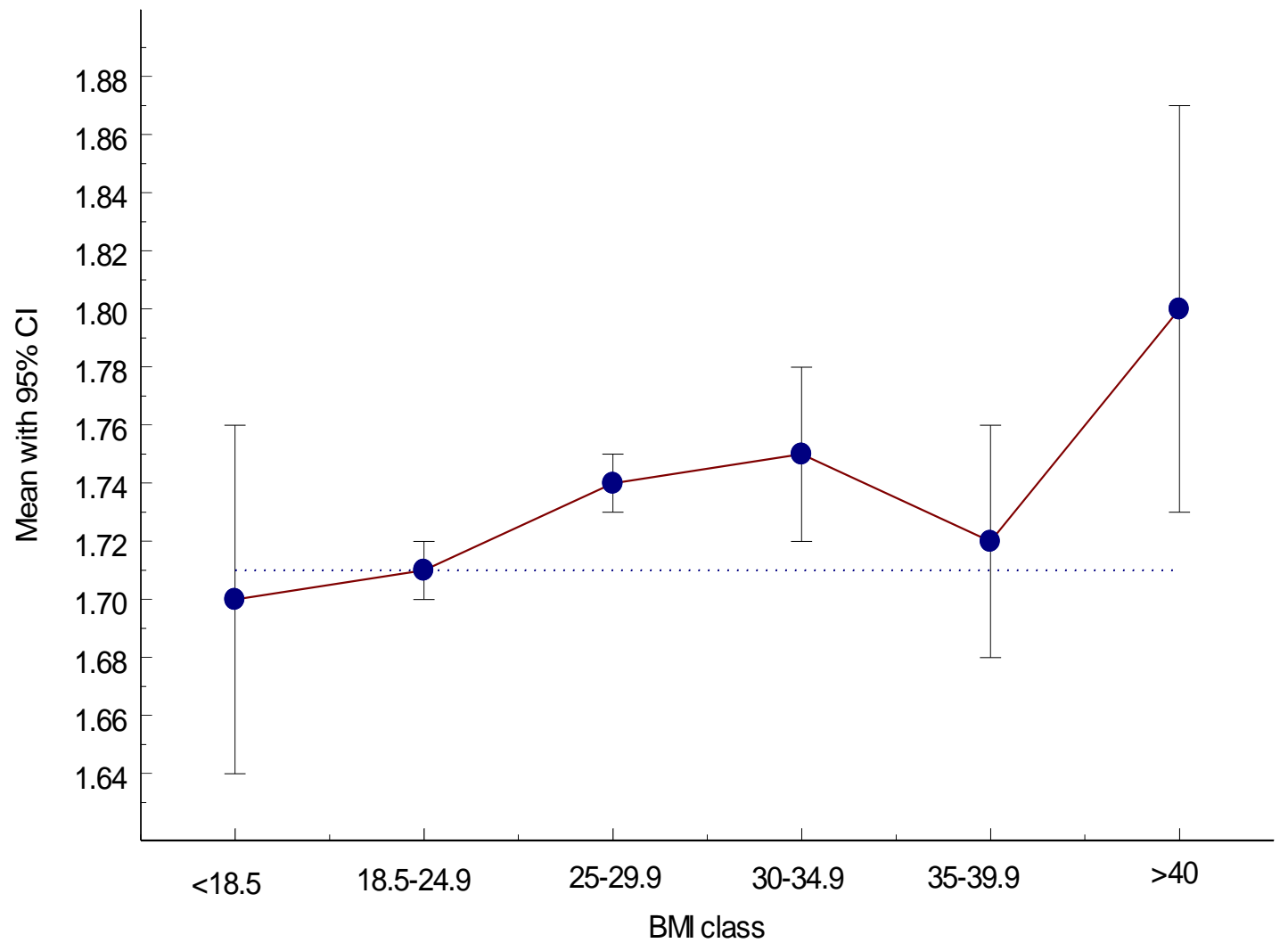


Figure 4: Means with 95\% CI of PAPP-A MoM and hCG MoM for different BMI classes based on study group II who had undergone prenatal screening. Horizontal lines show values from normal BMI (18.5-24.9).

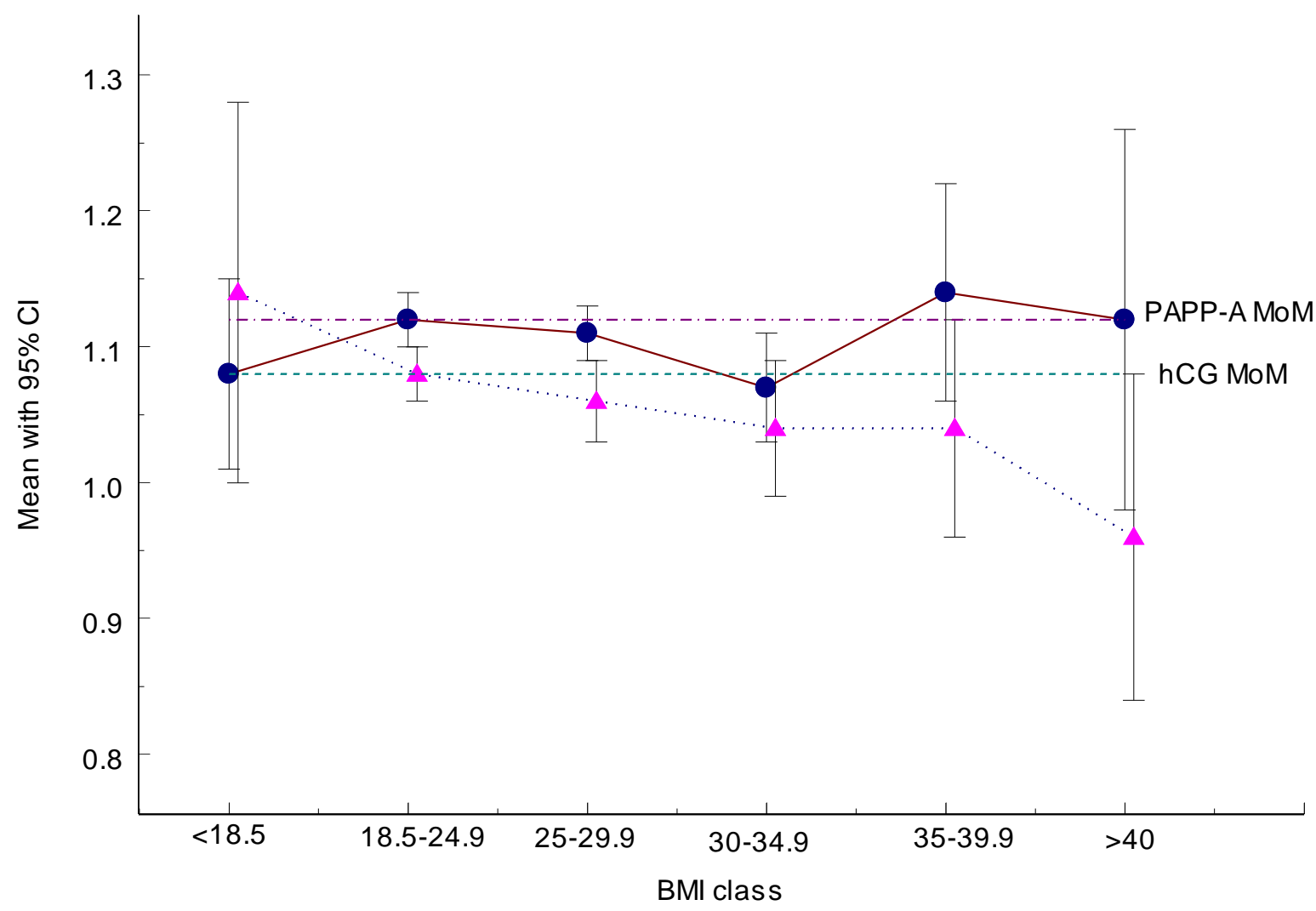


Figure 5: Mean trisomy 21 risk in the first trimester per 10,000 according to maternal age (1 year class) for different BMI classes (moving averages) based on study group II who had undergone prenatal screening.

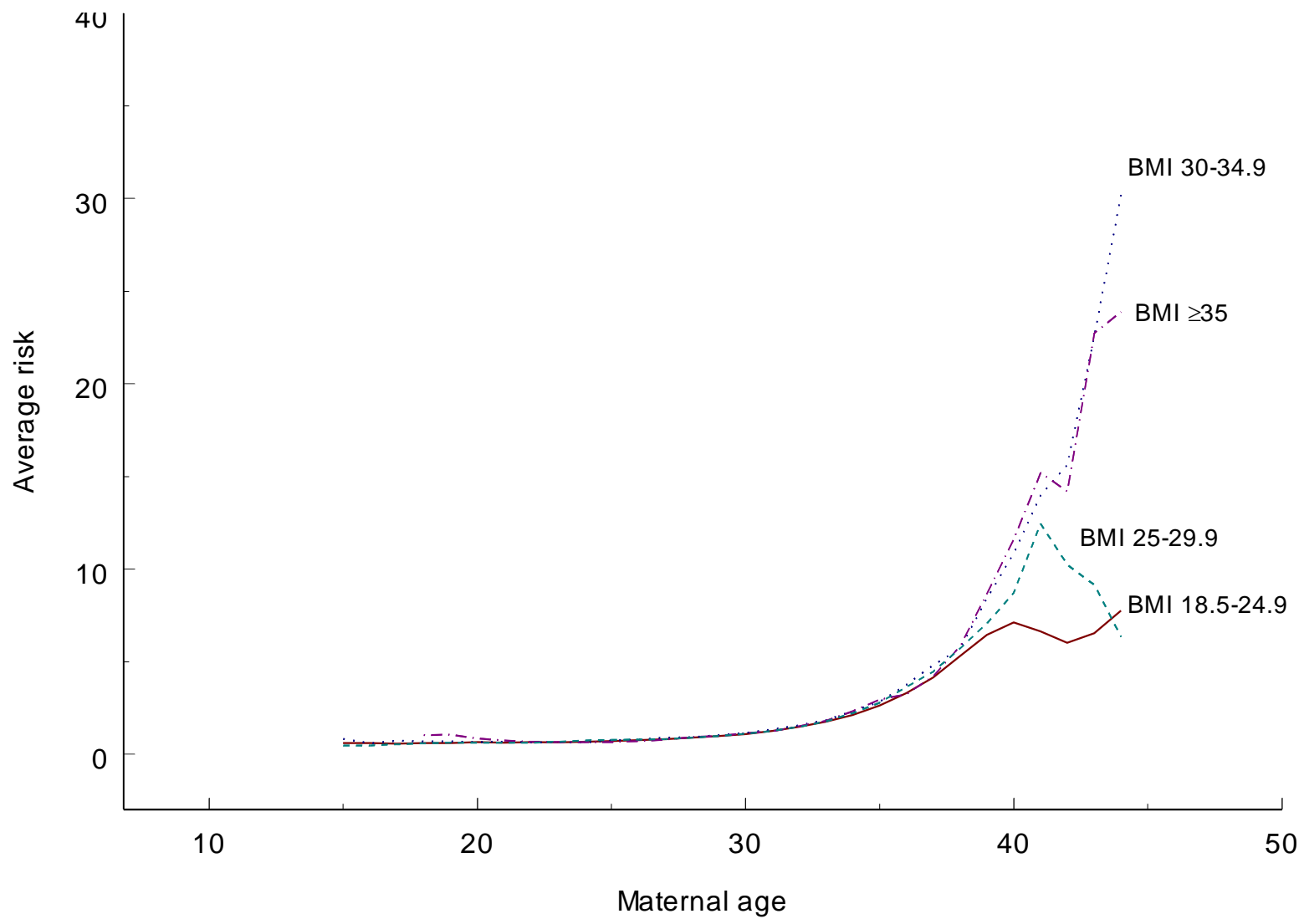

